\title{
The Impact of MMP-2 and Its Specific Inhibitor TIMP-2 Expression on the WHO Grade and Prognosis of Gliomas in Chinese Population: a Meta-Analysis
}

\author{
Guo-zhong $\mathrm{Yi}^{1} \cdot$ Wen-yan Feng ${ }^{2} \cdot$ Qiang Zhou $^{1} \cdot$ Ya-wei Liu ${ }^{1} \cdot$ Song-tao $\mathrm{Qi}^{1}$
}

Received: 25 June 2015 / Accepted: 9 November 2015 /Published online: 4 January 2016

(C) The Author(s) 2016. This article is published with open access at Springerlink.com

\begin{abstract}
So far, the prognostic value of matrix metalloproteinase 2 (MMP-2) and tissue inhibitor of matrix metalloproteinase 2 (TIMP-2) expressions in patients with gliomas has been widely reported, especially in China. But, the results were inconsistent. Thus, we conducted a meta-analysis to determine the correlation of MMP-2 and TIMP-2 expressions with the prognosis of patients with gliomas. Identical search strategies were used to search relevant literature in electronic databases updated to May 1, 2015, and odds ratios (ORs) with $95 \%$ confidence intervals ( $95 \%$ CIs) were estimated. Funnel plots and Egger's tests were conducted for the evaluation of publication bias, and heterogeneity and sensitivity were also analyzed. Finally, a total of 25 studies involving 1572 patients were included in the meta-analysis. Coincidentally, all these studies were conducted in Chinese population. It was found that MMP-2 expression was significantly associated with high-WHO grade gliomas $(n=24, \mathrm{OR}=6.54, \mathrm{CI}=4.98-8.60$; $\left.I^{2}=0 \%, P=0.911\right)$ and poor overall survival (OS), while it did not correlate to age $\left(n=2, \mathrm{OR}=0.78, \mathrm{CI}=0.35-1.74 ; I^{2}=\right.$ $0 \%, P=0.621)$ and gender $(n=2, \mathrm{OR}=1.15, \mathrm{CI}=0.51-$ $\left.2.62 ; I^{2}=0 \%, P=0.995\right)$. Moreover, the results of the pooled analysis indicated that there was no association between TIMP-2 expression and the WHO grade of gliomas $(n=7$,
\end{abstract}

Guo-zhong Yi and Wen-yan Feng contributed equally to this work.

Song-tao Qi

qisongtaosjwk@163.com

1 Department of Neurosurgery, Nanfang Hospital, Southern Medical University, Avenue North Road No.1838, Guangzhou 510515, People's Republic of China

2 The Second College of Clinical Medicine, Southern Medical University, Avenue North Road No.1838, Guangzhou 510515, People's Republic of China
$\left.\mathrm{OR}=1.02,95 \% \mathrm{CI}=0.68-1.54 ; I^{2}=71.4 \%, P=0.002\right)$, but the ratio of MMP-2 and TIMP-2 (MMP-2/TIMP-2) rose with the increase of the WHO grade of gliomas. In conclusion, there was no correlation between TIMP-2 expression and the WHO grade of gliomas, while MMP-2 expression was potently associated with high-WHO grade of gliomas.

Keywords MMP-2 - TIMP-2 · Gliomas · WHO grade · Prognosis $\cdot$ Meta-analysis

\section{Introduction}

Gliomas are the most common primary central nervous system tumor, whose treatment mainly comprises surgical resection, postoperative radiotherapy, and chemotherapy $[1,2]$. According to the World Health Organization, gliomas are divided into four clinical grades (I-IV) based on the morphology of histopathology [3]. While low-grade gliomas (I-II) are well differentiated, high-grade gliomas (III-IV) are undifferentiated and carry a worse prognosis $[4,5]$. Thus, it is indispensable to identify precise biomarkers with predictive value for the grading of gliomas and identification of the survival status of patients.

Matrix metalloproteinases (MMPs) are a family of enzymes which play a direct role in the tumorigenesis process [6]. Type IV collagen is the main component of extracellular matrix $(E C M)$ and basement membrane which form the first vital barrier in the course of tumor metastasis. Matrix metalloproteinase 2 (MMP-2), a main member of MMPs, by its ability to degrade the basement membrane type IV collagen, is thought to play a role in stromal and vascular invasion by tumor cells [7, 8]. Van Meter et al. reported that tissue inhibitors of MMPs (TIMPs) can block the action of MMPs and significantly decrease invasiveness of tumor [9]. And, TIMP2 which is a member of TIMPs has a unique dual function that 
being also an inhibitor of MMPs and with a more effective action on MMP-2 [10]. The levels of both MMP-2 and TIMP2 expressions in tumors may facilitate the initiation and progression of multiple biological behaviors required for tumor progression [10]. It has been identified that the expression of MMP-2 is associated with breast cancer [11], laryngeal cancer [12], and gastric cancer [13]. And, the amounts of TIMP-2 expression have also been found to be associated with different types of cancer $[14,15]$.

However, the results of studies on the association between MMP-2 or TIMP-2 expression and brain tumors were still contrary and there even existed conflicts. Some studies reported that high TIMP-2 expression was associated with high-grade gliomas [16], but some studies gave the contrary results [17], and others even showed that TIMP-2 expression was not correlated with the WHO grade of gliomas [18, 19]. Similarly, a number of studies investigated the impact of MMP-2 expression on the prognosis of patients with gliomas, but there were also no consistent results. In order to evaluate the essential roles and clinical utilities of MMP-2 and TIMP-2 in gliomas, we performed a meta-analysis.

\section{Materials and Methods}

\section{Search Strategy}

A literature search was carried out using PubMed, Ovid, EMBASE, Web of Science, China National Knowledge Infrastructure (CNKI), and Wanfang database updated to May 1, 2015. The search strategy used the following terms: ("metalloproteinase 2" or "MMP-2" or "type IV collagenase" or "gelatinaseA"), (" tissue inhibitor of metalloproteinase 2" or "TIMP-2"), "gliomas [MeSH]," and "prognosis." When the data reported in the articles were not enough for the analysis, the authors of articles were contacted. And, all references in retrieved articles were scanned to identify other potentially available studies.

\section{Inclusion and Exclusion Criteria}

Two reviewers (GZ. Yi and WY. Feng) independently selected eligible studies. Disagreements between the two reviewers were settled by discussion with the third reviewer (Q. Zhou). The inclusion criteria were as follows: (1) studies about MMP-2 or TIMP-2 expression in patients with gliomas; (2) case control or cohort studies; (3) the main outcome of studies concentrated on WHO grade, age, gender, and overall survival; (4) MMP-2 and TIMP-2 expression which was measured by immunohistochemistry (IHC); and (5) the hazard ratio (HR) or odds ratio (OR) value which could be obtained from the article directly.
Studies were excluded based on any of the following criteria: (1) review, editorial letters, comments, or nonhuman research and (2) lacked key information for HR or OR estimation analysis. For duplicated articles, only the one with the largest sample size or the most recent study was selected.

\section{Data Extraction and Quality Assessment}

The following information was gathered: the first author's name, year of publication, numbers of patients, mean age, WHO grade, MMP-2 and TIMP-2 expression assay methods, cutoff value of positive expression, and follow-up duration. Relevant data were extracted by two reviewers (GZ. Yi and WY. Feng) independently, and disagreement was resolved by the third reviewer (Q. Zhou).

The quality assessment of the included studies was conducted using the Newcastle-Ottawa Scale (NOS) with some modifications [20] (Table 1), which allowed for assessment of patient population and selection, comparability, and outcome. Total NOS scores ranged from 0 to 9 with a score $\geq 6$ indicating good quality [21].

\section{Statistical Analysis}

Quantitative meta-analysis was carried out using STATA version 12.0. Dichotomous data were presented as relative risks (RRs) or ORs, and continuous outcomes were presented as mean difference (MD), both with $95 \%$ confidence interval (CI). Cochrane's $Q$ test and $I^{2}$ measurement were performed to assess the heterogeneity, and heterogeneity was presented as significant when $I^{2} \geq$ $50 \%$ or $P \leq 0.10$. In the absence of statistical heterogeneity, a fixed-effect model was used to pool the results; otherwise, a random-effect model was used [22]. The effects of MMP-2 or TIMP-2 expression on pathological grade, age, and gender were considered as statistically significant if the corresponding $95 \%$ CI for each pooled OR did not overlap 1 , and an observed OR $>1$ indicated that gliomas of high-WHO grade were associated with high MMP-2 or TIMP-2 expression.

The potential risk of publication bias was examined by visual inspection of the funnel plots and was further assessed by Egger's linear regression test, which indicated the presence of a publication bias with $P \leq 0.10$. Sensitivity analysis was also conducted to evaluate the validity and reliability of the primary meta-analysis.

\section{Results}

\section{Characteristics of Studies}

A total of 241 articles were identified by computer and manual search. After further reading, we excluded 216 studies according to the eligibility criteria. Finally, 25 studies were included 
in the meta-analysis, of which 24 studies were related to MMP-2 and gliomas and seven studies for TIMP-2 and

Table 1 Newcastle-Ottawa quality assessment scale

\section{Selection}

1. Representativeness of the exposed cohort

(a) Truly representative of the average glioma patients in the community*

(b) Somewhat representative of the average glioma patients in the community*

(c) Selected group of users (e.g., nurses, volunteers)

(d) No description of the derivation of the cohort

2. Selection of the non exposed cohort

(a) Drawn from the same community as the exposed cohort*

(b) drawn from a different source

(c) No description of the derivation of the nonexposed cohort

3. Ascertainment of exposure (proof of gliomas and MMP-2 or TIMP-2 measurement)
(a) Secure record (e.g., surgical records)*
(b) Structured interview*
(c) Written self-report
(d) No description

4. Demonstration that outcome of interest was not present at start of study

(a) Yes*

(b) No

\section{Comparability}

1. Comparability of cohorts on the basis of the design or analysis

(a) Study controls for recurrence or metastasis*

(b) Study controls for any additional factor (age, gender, grade, etc.)*

Outcome

1. Assessment of outcome
(a) Independent blind assessment*
(b) Record linkage*
(c) Self-report
(d) No description

2. Was follow-up long enough for outcomes to occur? (death or recurrence)
(a) Yes (24 months)*
(b) No

3. Adequacy of follow-up of cohorts

(a) Complete follow-up - all subjects accounted for*

(b) Subjects lost to follow-up unlikely to introduce bias [small number lost (25\%) follow-up or description provided of those lost]*

(c) Follow-up rate $(<75 \%)$ and no description of those lost

(d) No statement

A maximum of one star $\left(^{*}\right)$ can be given for each numbered item within the "selection" and "outcome" categories, while a maximum of two stars (**) can be given for "comparability" gliomas. The flowchart of the study selection for the metaanalysis is shown in Fig. 1.

The major characteristics of all the included studies are summarized in Table 2. Interestingly, all the included studies were conducted in Chinese population, and the date of publication ranged from 2002 to 2014. Among all these studies, the correlation between the ratio of MMP-2 and TIMP-2 (MMP-2/TIMP-2) and the WHO grade of gliomas was offered in two studies [16, 39] and two studies [24, 27] provided the data of age and gender which were associated with the WHO grade of gliomas, and only one study [41] about MMP-2 expression reported the overall survival (OS) of patients. MMP-2 and TIMP-2 expressions in the tissues of gliomas were only investigated by IHC method. If the nucleus or cytoplasm was stained, the expression called local staining can be referred to as positive. For the cutoff value of positive expression, ten studies introduced the scored method according to percentage and intensity of stained cells, while the rest only used the percentage or intensity.

\section{MMP-2 Expression and Glioma Grade}

A total of 24 studies involving 1453 patients contained sufficient data for analyzing the association of MMP-2 expression with different WHO grades of gliomas. It was found that MMP-2 expression in patients with high-grade gliomas was

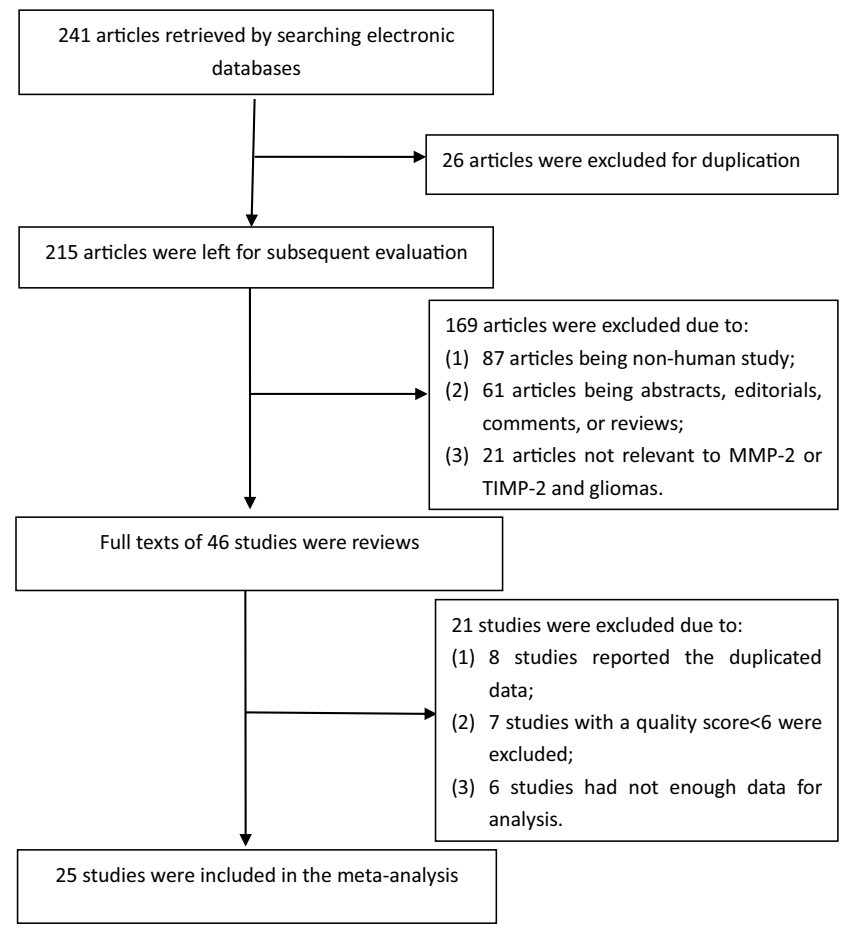

Fig. 1 Flow chart of study selection 
Table 2 Characteristics of included studies

\begin{tabular}{|c|c|c|c|c|c|c|c|c|c|}
\hline Study ID & $\begin{array}{l}\text { Sample size } \\
\text { (I-II/III-IV) }\end{array}$ & Mean age & Male/female & Method & $\begin{array}{l}\text { Cutoff of high } \\
\text { expression }\end{array}$ & $\begin{array}{l}\text { MMP-2 positive } \\
\text { (I-II/III-IV) }\end{array}$ & $\begin{array}{l}\text { TIMP-2 positive } \\
\text { (I-II/III-IV) }\end{array}$ & $\begin{array}{l}\text { Study quality } \\
\text { (points) }\end{array}$ & $\begin{array}{l}\text { Follow-up } \\
\text { (months) }\end{array}$ \\
\hline Sui R [23] & $120(70 / 50)$ & 54.6 & $82 / 38$ & IHC & Score $=1$ & $92(46 / 46)$ & NA & $7 / 9$ & NA \\
\hline Zeng ZQ [24] & $64(28 / 36)$ & 38.8 & $38 / 26$ & IHC & Score $=2$ & $43(15 / 28)$ & NA & $6 / 9$ & NA \\
\hline Guo GH [25] & $40(23 / 17)$ & 45.9 & $26 / 14$ & IHC & $5 \%$ & $24(9 / 15)$ & NA & $6 / 9$ & NA \\
\hline Wu HF [26] & $45(20 / 25)$ & 42.3 & $31 / 14$ & IHC & $10 \%$ & $21(5 / 16)$ & NA & $6 / 9$ & NA \\
\hline Pan LK [27] & $50(24 / 26)$ & 46.3 & $32 / 18$ & IHC & $10 \%$ & $37(14 / 23)$ & NA & $6 / 9$ & NA \\
\hline Li B [18] & $52(32 / 20)$ & 47.6 & $34 / 18$ & IHC & $10 \%$ & $22(9 / 13)$ & $23(15 / 8)$ & $6 / 9$ & NA \\
\hline Jv HG [16] & $78(43 / 35)$ & 42.4 & $48 / 30$ & IHC & Score $=1$ & $50(21 / 29)$ & $46(21 / 25)$ & $6 / 9$ & NA \\
\hline Liu Q [28] & $60(27 / 33)$ & 40.2 & $32 / 28$ & IHC & Score $=2$ & $49(19 / 30)$ & NA & $7 / 9$ & NA \\
\hline Sun SW [19] & $45(20 / 25)$ & 40.2 & $24 / 21$ & IHC & Score $=2$ & $36(13 / 23)$ & $24(9 / 15)$ & $6 / 9$ & NA \\
\hline Zhao YF [29] & $50(17 / 33)$ & 40.0 & $28 / 22$ & $\mathrm{IHC}$ & $10 \%$ & $34(8 / 26)$ & NA & $6 / 9$ & NA \\
\hline Song LJ [30] & $100(47 / 53)$ & 42.3 & $58 / 42$ & IHC & Score $=1$ & $76(24 / 52)$ & NA & $6 / 9$ & NA \\
\hline Kong LF [31] & $135(64 / 71)$ & 34.8 & $76 / 59$ & IHC & $5 \%$ & $49(11 / 38)$ & NA & $6 / 9$ & NA \\
\hline Lv ZH [32] & $30(12 / 18)$ & 41.3 & $12 / 18$ & IHC & $10 \%$ & $17(3 / 14)$ & NA & $6 / 9$ & NA \\
\hline Zhou R [17] & $67(27 / 40)$ & 43.1 & $40 / 27$ & IHC & $10 \%$ & $41(10 / 31)$ & $41(22 / 19)$ & $6 / 9$ & NA \\
\hline Li Hao [33] & $50(23 / 27)$ & 48.7 & $28 / 22$ & $\mathrm{IHC}$ & Local staining & $39(13 / 26)$ & NA & $6 / 9$ & NA \\
\hline Li Hong [34] & $119(55 / 64)$ & 42.0 & $66 / 53$ & IHC & Score $=1$ & NA & $106(45 / 61)$ & $7 / 9$ & NA \\
\hline Wang YT [35] & $45(20 / 25)$ & 40.2 & $21 / 22$ & $\mathrm{IHC}$ & Score $=1$ & $36(13 / 23)$ & NA & $6 / 9$ & NA \\
\hline Liu ZL [36] & $50(16 / 34)$ & 40.4 & $27 / 23$ & $\mathrm{IHC}$ & Score $=1$ & $46(13 / 33)$ & NA & $6 / 9$ & NA \\
\hline Tan YL [37] & $68(33 / 35)$ & 38.1 & $37 / 31$ & $\mathrm{IHC}$ & Score $=1$ & $49(16 / 33)$ & NA & $7 / 9$ & NA \\
\hline Shi QH [38] & $46(21 / 25)$ & 42.5 & $26 / 20$ & $\mathrm{IHC}$ & Local staining & $28(8 / 20)$ & NA & $6 / 9$ & NA \\
\hline Yi ZQ [39] & $46(22 / 24)$ & 38.5 & $26 / 20$ & $\mathrm{IHC}$ & $5 \%$ & $37(14 / 23)$ & $17(8 / 9)$ & $7 / 9$ & NA \\
\hline Li J [40] & $46(30 / 16)$ & 38.5 & $30 / 16$ & $\mathrm{IHC}$ & $5 \%$ & $20(7 / 13)$ & NA & $6 / 9$ & NA \\
\hline Xiao QH [41] & $60(20 / 40)$ & 35.0 & $42 / 18$ & $\mathrm{IHC}$ & $5 \%$ & $15(1 / 14)$ & NA & $8 / 9$ & $>24 \mathrm{M}$ \\
\hline $\begin{array}{l}\text { Luo GC [42] } \\
\text { Fu XW }\end{array}$ & $56(25 / 31)$ & 34.8 & $33 / 23$ & $\mathrm{IHC}$ & $5 \%$ & $47(18 / 29)$ & $42(22 / 20)$ & $6 / 9$ & NA \\
\hline$[43]$ & $50(25 / 25)$ & 48.5 & $27 / 23$ & IHC & $25 \%$ & $22(8 / 14)$ & NA & $6 / 9$ & NA \\
\hline
\end{tabular}

Tumor grade was described on the basis of the World Health Organization (WHO) grading system of primary brain tumors and divided into two groups: low grade (I-II) and high grade (III-IV)

IHC immunohistochemistry, NA not available

significantly higher than that in patients with low-grade gliomas $(n=24, \mathrm{OR}=6.54, \mathrm{CI}=4.98-8.60)$, and there was no significant heterogeneity in the meta-analysis $\left(I^{2}=0 \%, P=\right.$ 0.911) (Fig. 2). And, no correlation was observed between MMP-2 expression and age $(n=2, \mathrm{OR}=0.78, \mathrm{CI}=0.35-1.74$; $\left.I^{2}=0 \%, P=0.621\right)$ and also between MMP-2 and gender $\left(n=2, \mathrm{OR}=1.15, \mathrm{CI}=0.51-2.62 ; I^{2}=0 \%, P=0.995\right)$ through a fixed-effect model (Fig. 3). The association of MMP-2 expression with overall survival (OS) was reported in one study [41], and the results showed that high MMP-2 expression was significantly associated with poor OS, while the exact data were not reported.

\section{TIMP-2 Expression and Glioma Grade}

There were seven studies involving 463 patients on TIMP-2 expression and the WHO grade of gliomas. The results of pooled analysis demonstrated that there was no significant association between TIMP-2 expression and tumor grade $(n=7, \mathrm{OR}=1.02,95 \% \mathrm{CI}=0.68$ 1.54) (Fig. 4a), but there was significant heterogeneity $\left(I^{2}=71.4 \%, P=0.002\right)$, so we performed the analysis through a random-effect model. And, among these seven studies involved, there were six studies that also reported MMP-2 expression and glioma grade, so we also conducted a meta-analysis to investigate the association between MMP-2 expression and glioma grade among these six studies. However, the overall trend was not changed $\left(n=6, \mathrm{OR}=5.79,95 \% \mathrm{CI}=3.36-9.98 ; I^{2}=\right.$ $0 \%, P=0.982$ ) (Fig. 4b).

\section{MMP-2/TIMP-2 and Glioma Grade}

Two studies reported the association between MMP-2/ TIMP-2 and the WHO grade of gliomas. Of which, one study [16] used the percentage of positive cells after 


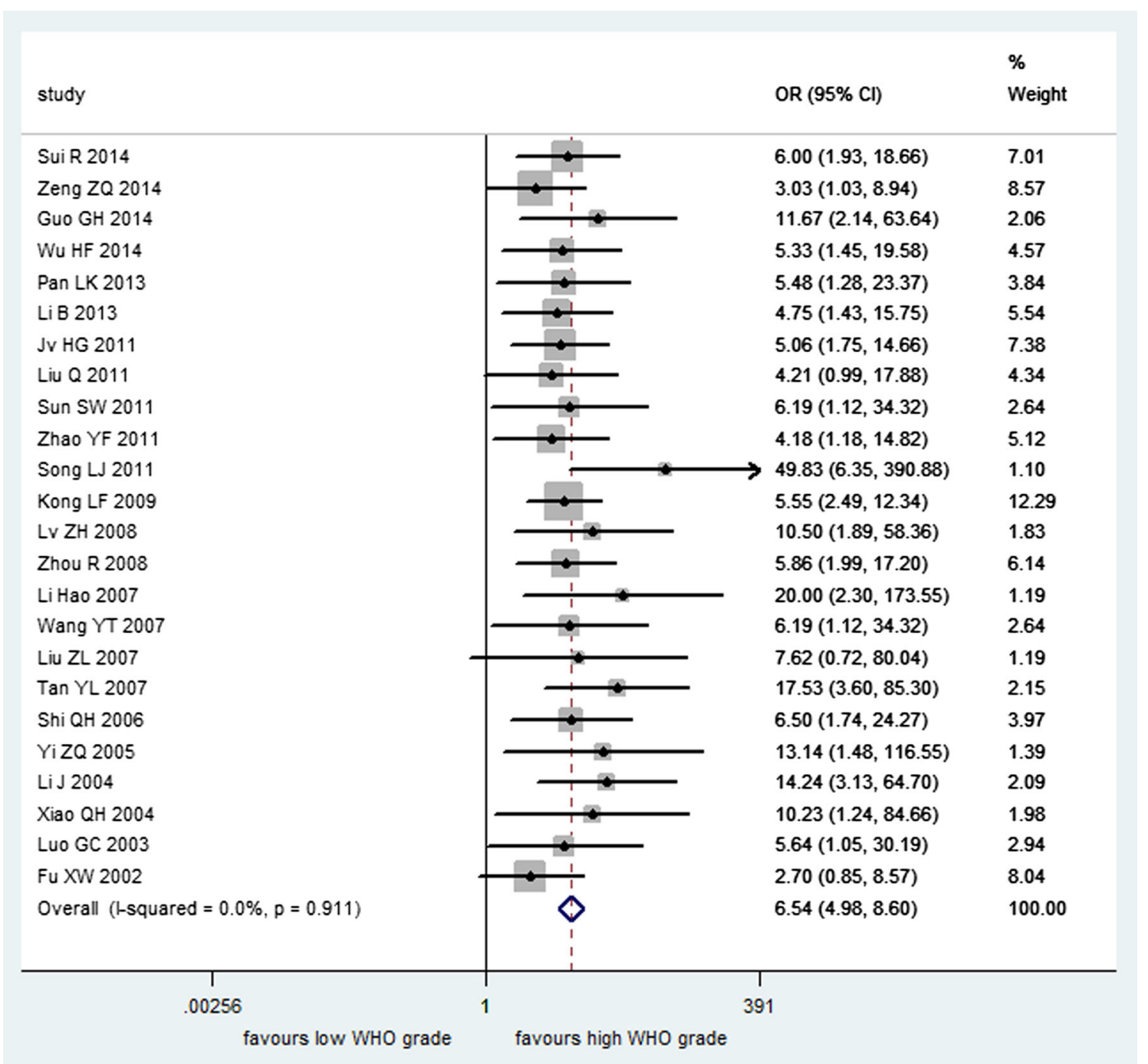

Fig. 2 Forest plot of association between MMP-2 expression and the WHO grade of gliomas

IHC to calculate the ratio, and the results showed that with the increase of tumor grade, MMP-2/TIMP-2 rose from 1.00 to 1.18. The other study [39] detected the gray value of microscopic field $(10 \times 40)$ and calculated the ratio, which the reported ratio is $(0.83 \pm 0.046)$ for low-grade gliomas and $(0.46 \pm 0.094)$ for high-grade tumor, while a high gray value represented the lower percentage of positive cells.

\section{Publication Bias and Sensitivity Analysis}

Visual inspection of the funnel plot revealed asymmetry in analysis of the association between MMP-2 or TIMP-2 expression and glioma grade, indicating the possibility of publication bias (Fig. 5a, b). We also estimated the publication bias through using Egger's linear regression test. The results revealed support for significant publication bias in MMP-2 group $(t=3.90, P=0.001)$ while did not reveal any publication bias among studies on TIMP-2 expression and glioma grade $(t=-0.69, P=0.521)$.

Sensitivity analyses were also conducted to ascertain the effects attributable to any individual study, while the results showed that no individual study could change the overall trends. It suggested that the results of the meta-analysis were stable (Fig. 5c, d).

\section{Discussion}

Gliomas are the most common type of primary cerebral tumors. According to the WHO, gliomas can be divided into four clinical grades [3]. However, the molecular functions in glioma grade are still not wholly understood, and the prognoses of patients with gliomas remain dismal [44]. It is important for us to find the molecular markers for the progression and prognosis of gliomas, which would be of great benefit in selecting the 


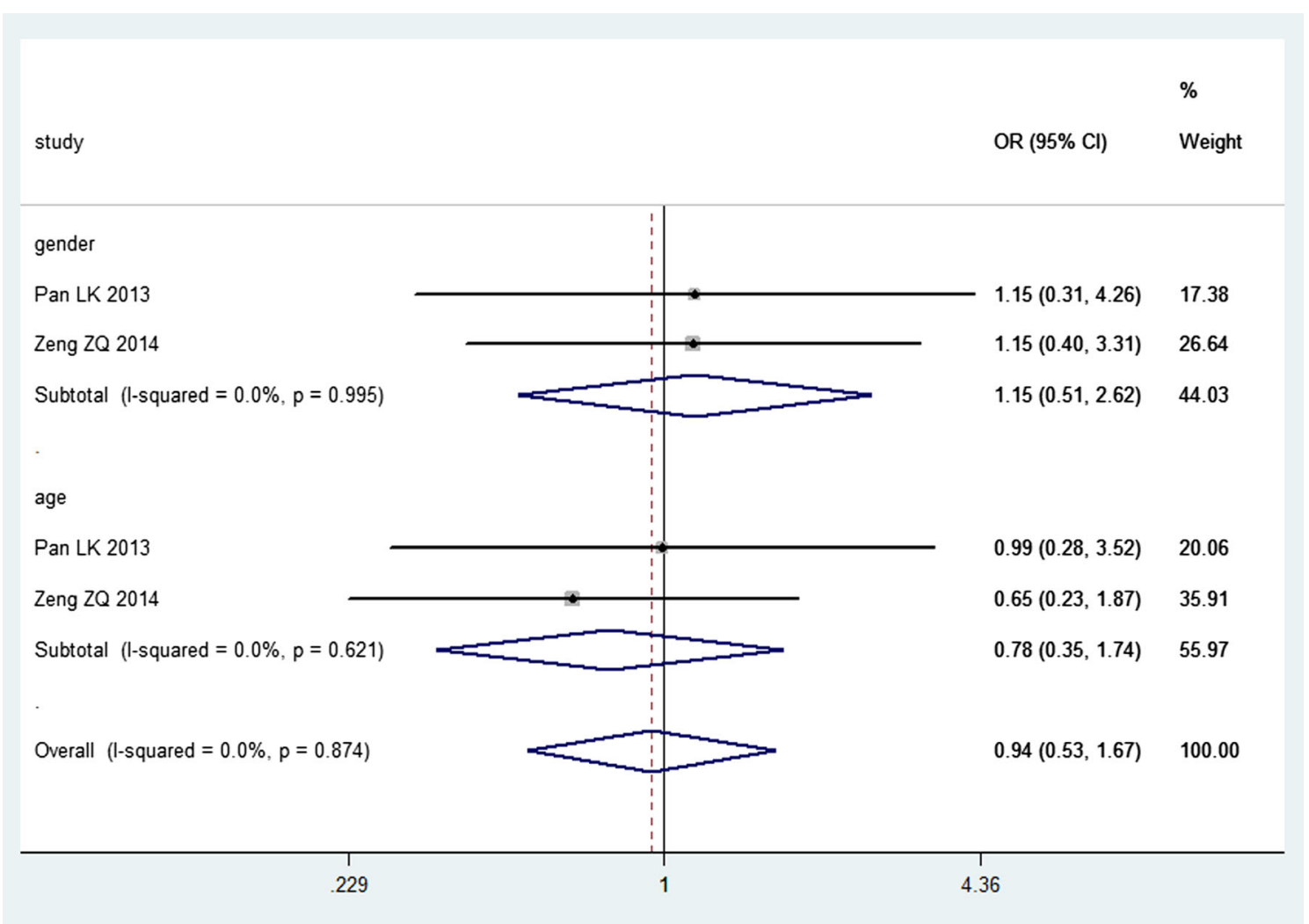

Fig. 3 Forest plot of association between MMP-2 expression and gender, age

therapeutic strategies and improving patients' survival. Many studies have shown that MMP-2 and TIMP-2 play crucial roles in various human cancers, including gliomas. But, all the results of these studies about gliomas remained controversial, so it is necessary to perform a meta-analysis.

In our meta-analysis, the results of 24 studies involving 1453 patients demonstrated that the expression of MMP- 2 in patients with high-grade gliomas increased significantly as compared to patients with low-grade gliomas, while no correlation was observed between MMP-2 and age, and also no association was found between MMP-2 and gender. There were also evidences that high MMP-2 expression was associated with poor OS in patients with gliomas. Moreover, our meta-analysis determined that there was no association between TIMP-2 expression and the WHO grade of gliomas, and the overall trend of MMP-2 expression and glioma grade was not changed in studies which have reported both MMP-2 and TIMP-2 expressions. And, MMP-2/TIMP-2 rose with the increase of glioma grade, which may be used as criteria of WHO grade in gliomas.
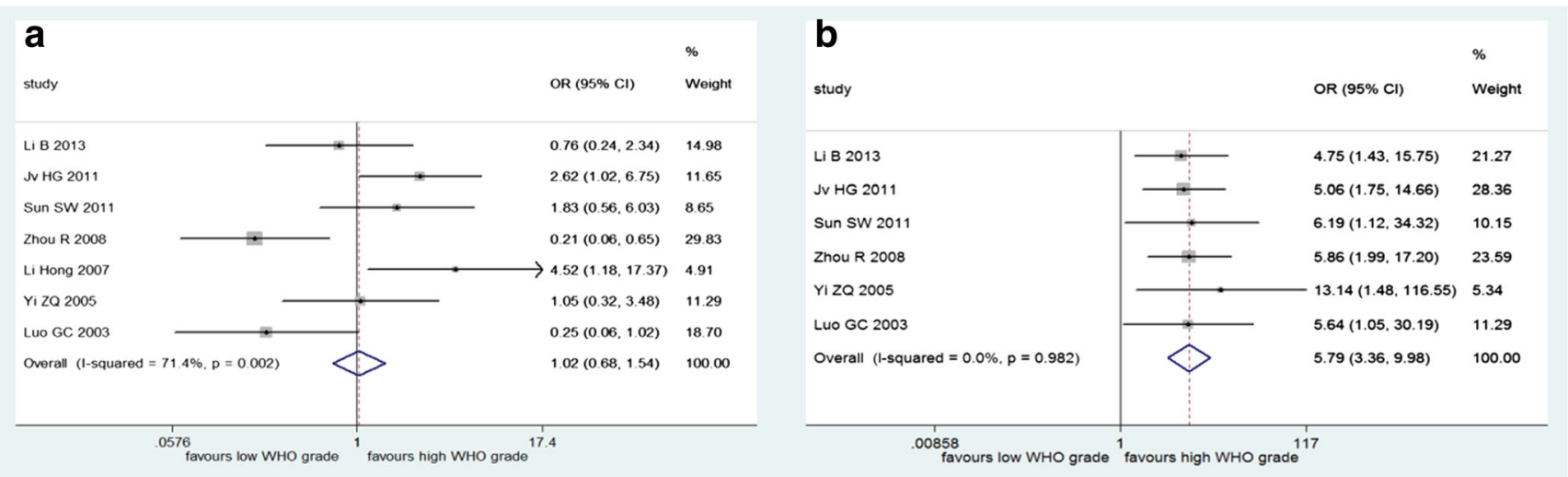

Fig. 4 Forest plot of association between TIMP-2 expression and the WHO grade of gliomas (a) and association between MMP-2 expression and glioma grade in studies which have also reported TIMP-2 expression (b) 

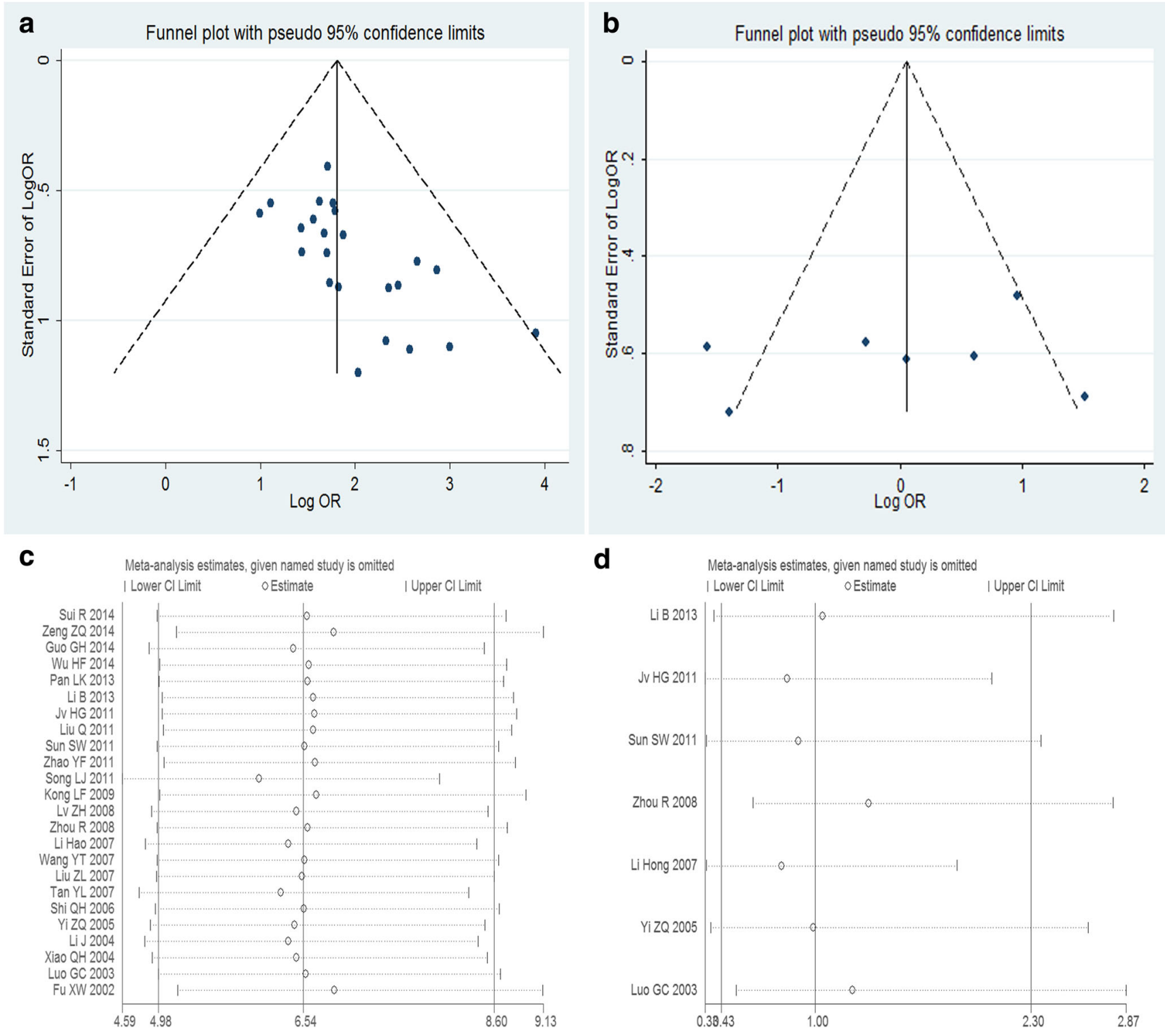

Fig. 5 Funnel plots and sensitivity analysis of the meta-analysis. Funnel plots of the meta-analysis assessing a MMP-2 expression and glioma grade and b TIMP-2 expression and glioma grade and sensitivity

Both funnel plots and Egger's tests indicated that publication biases were present in the analysis of association of MMP-2 expressions with glioma grade, though we performed a quality assessment of studies to avoid some selection biases and attempted to minimize publication bias by performing the literature search as complete as possible. However, our search was restricted to studies published in English or Chinese, and the studies with negative results were often rejected [45]. So, publication bias may be a limitation in our meta-analysis.

Despite our efforts to conduct a comprehensive analysis, there were also some other limitations which should be discussed. Firstly, the heterogeneity among studies about TIMP-2 expression and glioma grade may affect the results

analysis of the meta-analysis assessing $\mathbf{c}$ MMP-2 expression and glioma grade and $\mathbf{d}$ TIMP-2 expression and glioma grade

of the present meta-analysis. Secondly, all the MMP-2 and TIMP-2 expressions were measured by IHC and all the studies were conducted in Chinese population. IHC was the most frequently applied method, but methodological differences may contribute to the heterogeneity. And, subgroup analysis could not be conducted to address these technical problems because few studies offered the concrete data. Thirdly, there was no consistent threshold value to define positive expression in assessment of MMP-2 and TIMP-2 expressions, which can be the cause of potential bias. Lastly, there was only one study that reported the data about MMP-2 expression and the OS and only two studies analyzed the association between MMP-2/TIMP-2 and the grade of gliomas. Due to the limited number of studies included, the findings about the impact of 
MMP-2 expression or MMP-2/TIMP-2 on the prognosis of gliomas should be interpreted with caution.

Considering all these limitations existing in this meta-analysis, the conclusions of this meta-analysis should be drawn carefully. In summary, our meta-analysis concluded that high MMP-2 expression was associated with high-grade gliomas, and there was no correlation between TIMP-2 expression and the WHO grade of gliomas. MMP-2/TIMP-2 testing may predict the WHO grade of gliomas, and MMP-2 expression may serve as a biomarker for the prognosis of patients with gliomas, which required to be further certified by future studies.

Acknowledgments This study was supported by Key Clinical Specialty Discipline Construction Program and the National Natural Science Foundation of China (Grant Nos. 81572498, 81472315, 81372692).

\section{Compliance with Ethical Standards}

Conflict of Interest All the authors declare that there is no conflict of interest.

Open Access This article is distributed under the terms of the Creative Commons Attribution 4.0 International License (http:// creativecommons.org/licenses/by/4.0/), which permits unrestricted use, distribution, and reproduction in any medium, provided you give appropriate credit to the original author(s) and the source, provide a link to the Creative Commons license, and indicate if changes were made.

\section{References}

1. Woolf EC, Scheck AC (2012) Metabolism and glioma therapy. CNS Oncol 1(1):7-10

2. Mack F, Schfer N, Kebir S, Stuplich M, Schaub C, Niessen M, Scheffler B, Herrlinger U et al (2014) Carmustine (BCNU) plusteniposide (VM26) in recurrent malignant glioma. Oncology 86(5-6):369-372

3. Louis DN, Ohgaki H, Wiestler OD, Cavenee WK, Burger PC, Scheithauer BW, Jouvet A, Kleihues P (2007) The 2007 WHO classification of tumours of the central nervous system. Acta Neuropathol 114(2):97-109

4. Kleihues P, Louis DN, Scheithauer BW, Rorke LB, Reifenberger G, Burger PC, Cavenee WK (2002) The WHO classification of tumors of the nervous system. J Neuropathol Exp Neurol 61(3):215-225

5. Ahmed R, Oborski MJ, Hwang M, Lieberman FS, Mountz JM (2014) Malignant glioma: current perspectives in diagnosis, treatment, and early response assessment using advanced quantitative imaging methods. Cancer Manag Res 6:149-170

6. Ray JM, Stetler-Stevenson WG (1994) The role of matrix metalloproteases and their inhibitors in tumour invasion, metastasis and angiogenesis. Eur Respir J 7(11):2062-2072

7. Hojilla CV, Mohammed FF, Khokha R (2003) Matrix metalloproteinases and their tissue inhibitors direct cell fate during cancer development. Br J Cancer 89(10):1817-1821

8. Stamenkovic I (2003) Extracellular matrix remodelling: the role of matrix metalloproteinases. J Pathol 200(4):448-464

9. Van Meter T, Rooprai HK, Rucklidge GJ, Pilkington GJ (1997) Functional blocking with TIMP-1 and anti-alpha-V integrin: evidence for cooperation of MMPs and integrins in glioma invasion in vitro. Anticancer Res 17:1051
10. Bourboulia D, Stetler-Stevenson WG (2010) Matrix metalloproteinases (MMPs) and tissue inhibitors of metalloproteinases (TIMPs): positive and negative regulators in tumor cell adhesion. Semin Cancer Biol 20:161-168

11. Chen Y, Wang X, Chen G, Dong C, Zhang D (2015) The impact of matrix metalloproteinase 2 on prognosis and clinicopathology of breast cancer patients: a systematic meta-analysis. PLoS One 10(3): 0121404

12. Liu RR, Li MD, Li T, Tan Y, Zhang M, Chen JC (2015) Matrix metalloproteinase-2 (MMP-2) protein expression and laryngeal cancer prognosis: a meta-analysis. Int J Clin Exp Med 8(2):2261-2266

13. Donizy P, Rudno-Rudzinska J, Kaczorowski M, Kabarowski J, Frejlich E, Kielan W, Matkowski R, Halon A (2015) Disrupted balance of MMPs/TIMPs in gastric carcinogenesis-paradoxical low MMP-2 expression in tumor and stromal compartments as a potential marker of unfavorable outcome. Cancer Investig. doi:10. 3109/07357907.2015.1024316

14. Alakus H, Grass G, Hennecken JK, Bollschweiler E, Schulte C et al (2008) Clinicopathological significance of MMP-2 and its specific inhibitor TIMP-2 in gastric cancer. Histol Histopathol 23(8):917923

15. Ross JS, Kaur P, Sheehan CE, Fisher HA, Kaufman RA Jr et al (2003) Prognostic significance of matrix metalloproteinase 2 and tissue inhibitor of metalloproteinase 2 expression in prostate cancer. Mod Pathol 16(3):198-205

16. Jv HG, Jiang JY, Shen SP, Geng H (2010) Expressions of CD147, MMP-1, MMP-2, MMP-9, TIMP-1, TIMP-2 in different grades gliomas patient. Journal of TAISHAN medical college 31(9):653-655

17. Zhou R, Bai LN, Wang RZ, Zhang LZ, Bao XJ, Cao J (2008) Expression of MMP-2 and its inhibitor TIMP-2 in human glioma. Journal of Xi'an Jiao tong University (Medical Sciences) 29(6): 686-688, 701

18. Li B, Yi L, Xu LS, Xu MH (2013) Analysis of MMP-1, MMP-2, MMP-9 and TIMP-1, TIMP-2 detection in diagnosis of glioma. Chin J Pract Nerv Dis 16(23):4

19. Sun SW, Wang YT, Wang DK, Liu Q, Zang ZX (2011) Study of MMP-2, MMP-9, TIMP-1 and TIMP-2 expression in human glioma. Acta Acad Med Weifang 33(1):16-18

20. Wells G SB, O'Connell D. The Newcastle-Ottawa Scale (NOS) for assessing the quality of nonrandomised studies in meta-analyses. Ottawa Health Research Institute Web site. http://www.ohri.ca/ programs/clinical_epidemiology/oxford.asp. Accessed 26 May 2015

21. Stang A (2010) Critical evaluation of the Newcastle-Ottawa scale for the assessment of the quality of nonrandomized studies in metaanalyses. Eur J Epidemiol 25(9):603-605. doi:10.1007/s10654010-9491-Z

22. J. Higgins and S. Green, Cochrane Handbook for systematic reviews of interventions, Version 5.1.0, he Cochrane Collaboration, 2011, http://handbook.cochrane.org/. Accessed 26 May 2015

23. Sui R, Zhang Y, Chen Y, Pao HZ (2014) The valuation of combined detection of MMP-2 and MMP-9 in diagnosis of glioma. Chin J Lab Diagn 18(2):205-206

24. Zeng ZQ, Liu H (2014) Expression of macrophage migration inhibitory factor and matrix metalloproteinase 2 in glioma tissue and its clinical significance. PJCCPVD (7):44 45, 53

25. Guo GH, Zhuang YJ, Qiu H, Li YC, Chen G, Yu XD, Wang YJ (2014) The significance of galectin-3 and matrix metalloproteinase-2 expression in patients with glioma. Clin Med China 7:735-738

26. Wu HF, Fu BM (2014) The expression and significance of Ang-2 and MMP-2 in glioma. Chin J Pract Nerv Dis (6):77-78, 79

27. Pan LK, Liu HY, Gao JZ, Guo YF, Chang XP, Dong CY (2013) Expression and significance of PTTG, MMP-2 and Survivin in glioma. Mod Oncol 21(8):1730-1732 
28. Liu Q, Li G, Wang DK, Cao PC, Wang YH (2011) The clinical significance and expression of MMP-2 and MMP-9 in glioma. Chin J Gerontol 31(16):3032-3033

29. Zhao YF, Liu S, Lu DS (2011) The correlation between the expression of MMP-2, VEGF and the malignancy of glioma. Chongqing Med J 40(13):1316-1318

30. Song LJ, Ma SQ, Guo FY, Sun HW, Wang RY (2011) Expression and significance of activating transcription factor 3, mammary serine protease inhibitor and matrix metalloproteinase 2 in human gliomas. Chin J Brain Dis Rehabil 01(1):4-10

31. Kong LF (2009) The study on significance and detection of PRL-3 and MMPs in human gliomas. The Fourth Military Medical University, Dissertation

32. Lv ZH, Zhang XX, Zhang R, Li RY (2008) Expression and significance of MDM2, p15, MMP-2 proteins in the glioma. Mod Oncol 16(7):1127-1129

33. Li H, Chen J, He M, Hui XH, Cai BW, Li Y (2007) Expression of CD147 and MMP-2 in human gliomas and its correlations with prognosis. J Sichuan Univ 38(3):396-399

34. Li Hong (2007) The expression of TIMP-1 and MMP-2 in gliomas. Dissertation, University of Qingdao

35. Wang YT, Liu Q, Wang CD, Wang DK, Zhang ZX (2007) Relationship between expression of matrix metalloproteinase-2 and clinical pathology in glioma. Acta Acad Med Weifang 29(2): $126-127$

36. Liu ZL, Zhang S, Zhang WB, Ren DP (2007) The expression of MMP-2, MMP-9 and PCNA in human gliomas. Chin J Ner Ment Dis. 33(9):532-536, 547
37. Tan YL, Fang C, Hu ZJ, LIU SZ, Yang X, Wu YP (2007) Expression of COX-2 in human brain glioma and the relation to invasion of the tumor. J Hebei Med Coll Continuing Educ 24(1):1-3

38. Shi QH, Zhi XG (2006) Expression of CD147 and its relationship with the expression of MMP-2 in glioma. Acta Academiae Medicinae Jiangxi 46(1):19-22

39. Yi ZQ, Hou RG, Wang T, Wang MD (2005) The expression and significance of MMP-2 and TIMP-2 in gliomas. Chin J Neuromed 4(11):1097-1100

40. Li J, Ye YH (2004) Expression and significance of MMP-2 and integrin $\beta 1$ in human gliomas. Cancer Res Prev Treat 31(3):138-139

41. Xiao QH (2004) PDGFR $\alpha$, MMP-2, MMP-9 and p53 Expression is associated with the infiltrative nature and prognosis of glioma. Dissertation, University of Sichuan

42. Luo GC, Ye YH (2003) Expressions of matrix metalloproteinase-2 and tissue inhibitor of metalloproteinase-2 in human brain glioma and their significances. Chin J Clin Neurosurg 8(4):283-285

43. Fu XW, Jiao BH, Zhang XH (2002) Expression and clinical significance of E-cd, MMP-2 and CD44v6 gene in glioma. Mod J Neurol Neurosurg 2(1):40-43

44. Wen PY, Kesari S (2008) Malignant gliomas in adults. N Engl J Med 359(8):492-507

45. Begg CB, Berlin JA (1988) Publication bias: a problem in interpreting medical data. J R Stat Soc A 81(2):107-115 